Obada B²., Serban Al. O. ${ }^{1}$

\title{
Achilles tendon ruptures: comparison between the clinical results of classical versus mini-invasive or percutaneous surgical treatment
}

${ }^{1}$ Clinic of Orthopedics and Traumatology, Emergency County Hospital Constanța

${ }^{2}$ Faculty of Medicine, "Ovidius" University of Constanta, Romania

\begin{abstract}
The aim of the study is to evaluate the surgical treatment comparing the results obtained with different techniques. We revised 68 cases with acute Achilles tendon rupture who underwent surgical correction between 2004 and 2011 , with a 40 month average follow-up. 34 of these were submitted to a classical open repair using the Kessler or Krakow technique, 25 to a mini-invasive technique (Achilon) and 9 to a percutaneous technique (Tenolig). We report a 29\% rate of complications when using the classical technique: the major complications were one re-rupture, two surgical wound dehiscences, one infection and one sural nerve injury. In the miniinvasive/percutaneous techniques, two re-ruptures occurred (5.9\% total, one in each technique) and one fistula at the needle insertion location. In regards to the percutaneous and mini-invasive techniques, the functional results and degree of satisfaction were higher, with fewer complications, reflecting a trend that has been expressed in the international literature.
\end{abstract}

Keywords: Achilles, tendon, rupture, mini-invasive, percutaneous, classical surgery

\section{Bogdan Obada}

Email: bogdanobada@yahoo.com

\section{Introduction}

Acute Achilles tendon ruptures occur mostly in male patients, between 30 and 50 years of age, frequently associated with sports practice. $[1,2]$. The aim of the study is to evaluate the surgical treatment comparing the results obtained with different techniques.

\section{Material and Methods}

We revised 68 cases with acute Achilles tendon rupture who underwent surgical correction between 2004 and 2011, with a 40 month average follow-up. 34 of these were submitted to a classical open repair using the Kessler or Krakow technique, 25 to a miniinvasive technique (Achilon) and 9 to a percutaneous technique (Tenolig). $[3,4,5]$ (Figure1).

In each patient, we determined the mechanism of injury, surgical and inpatient time, surgical technique, complications and re-rupture rate. The 

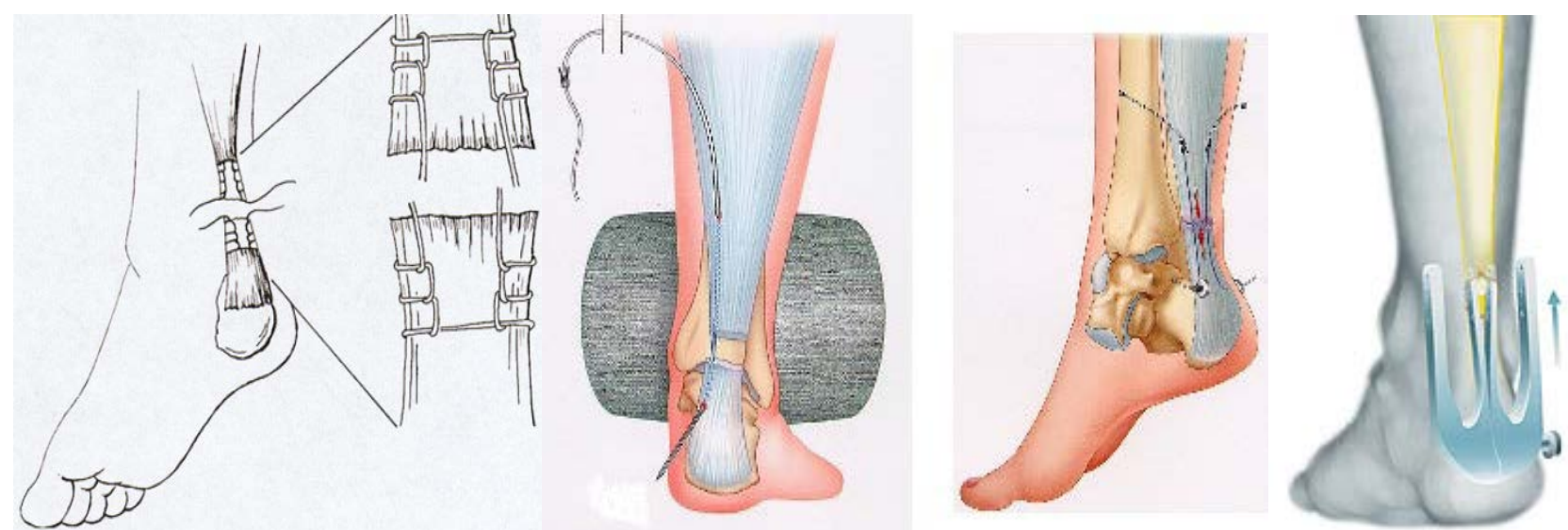

Figure 1 - Surgical techniques for Achilles tendon repair: Krakow, Tenolig, Achillon techniques

AOFAS hindfoot score were used for clinical mini-invasive (94) techniques.(Table I) and functional analysis (Figure2). Limitations in daily living and sports activities and global patient satisfaction were reviewed.

Nearly all patients fully resumed their activities of daily living (95.6\%) and $73.5 \%$ denied limitations is sports practice.

\section{Results}

\section{Discussions}

On the 68 cases, 93\% were male, with an average age of 38.7 years $(18,55)$. The average time to surgery was 1.53 days and average inpatient time was 2.9 days. The most frequent cause of injury was sports practice $(47 \%)$. The AOFAS average score was 89.8 points $(89,100)$ for the classical technique, and 93.9 points $(80,1000)$ for the percutaneous $(93.5)$ and
We report a $29 \%$ rate of complications when using the classical technique: the major complications were one re-rupture, two surgical wound dehiscences, one infection and one sural nerve injury. In the miniinvasive/percutaneous techniques two re-ruptures occurred (5.9\% total, one in each technique) and one fistula at the needle insertion location (Figure 3).

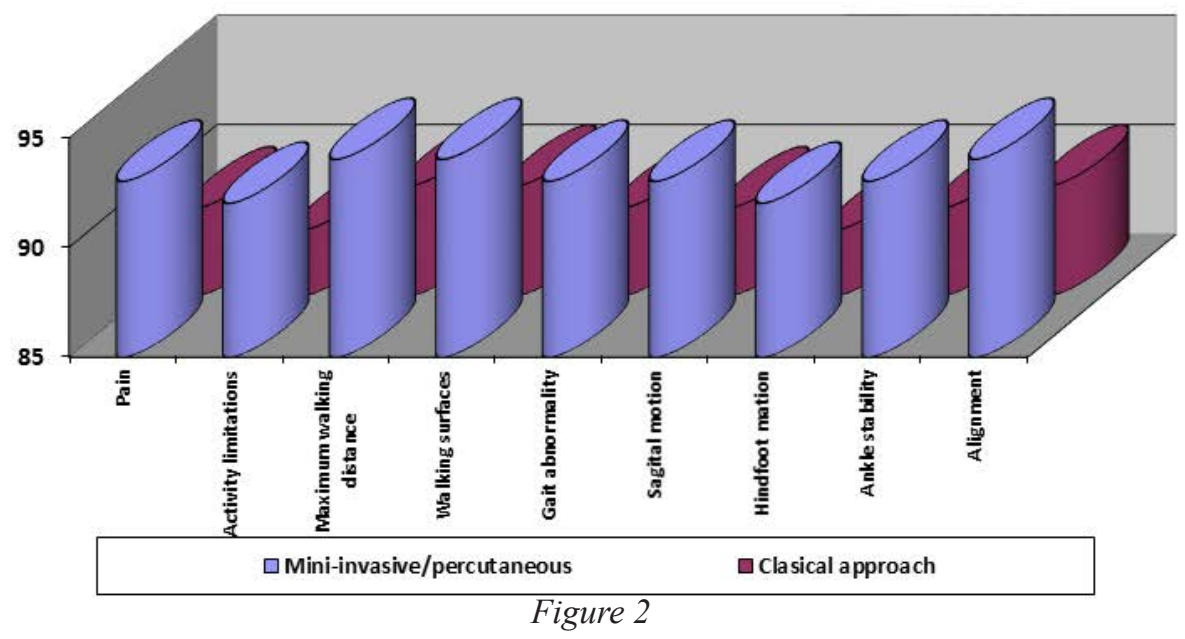




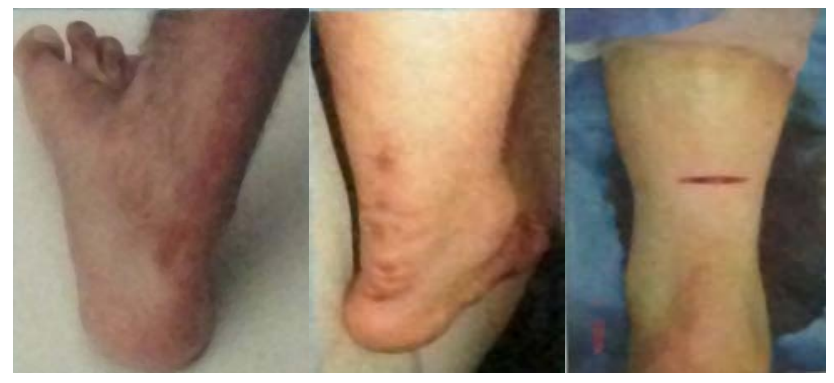

Figure 3 - Clinical aspects

After casting, rehabilitation is important to increase the functionality of the ankle and to return the patient to his social life. There is a tendency for a higher rate of return to sports activities at the same level to be achieved after classical treatment or miniinvasive or percutaneous treatment of an Achilles tendon rupture $[6,7]$. Patients who return to sports activities are $78.93 \%$ from the patients with miniinvasive or percutaneous treatment and $82.75 \%$ from those with classical surgical treatment and we believe that our indications and approaches of Achille tendons rupture are as good as we see in literature. hand, a slightly higher re-rupture rate. We were not able to obtain, however, a statistically significant correlation between the functional scores regarding the percutaneous when compared separately with the mini-invasive or classical techniques (explainable by the reduced sample of the percutaneous technique). Similarly, there was no statistically significant correlation between age, gender, side of intervention, mechanism of injury, pre-operatory time or return to previous activities and the rate of complications.

In regards to the percutaneous and miniinvasive techniques, the functional results and degree of satisfaction were higher, with fewer complications, reflecting a trend that has been expressed in the international literature.

Presently, in ourdepartment, mostacuteAchilles tendon ruptures are treated using a percutaneous or mini-invasive technique, as these techniques seem to be associated with a lower complication rate and better functional results.

\section{References}

\section{Conclusions}

The non-classical techniques were associated with a lower rate of complication when compared to the classical technique, whilst having, on the other
1. Leppilahti, J. (1996). Achilles tendon rupture, with special reference to epidemiology and results of surgery (dissertation), University of Oulu

2. Zollinger, H., Rodniguez, M. \& Genoni, M. (1983). Zur Atiopathogenese und Diagnostik der

Table I

\begin{tabular}{|c|c|c|c|}
\hline & Classical approach & $\begin{array}{l}\text { Mini-invasive } \\
\text { technique (Achillon) }\end{array}$ & $\begin{array}{l}\text { Percutaneous } \\
\text { technique (Tenolig) }\end{array}$ \\
\hline \multirow{2}{*}{ Number of patients } & \multirow[t]{2}{*}{34} & 25 & 9 \\
\hline & & \multicolumn{2}{|l|}{34} \\
\hline \multirow[t]{2}{*}{$\begin{array}{l}\text { Average } \\
\text { Score }\end{array}$} & \multirow[t]{2}{*}{$\begin{array}{l}89.8 \\
(80,100)\end{array}$} & $\begin{array}{l}94 \\
(80,100)\end{array}$ & $\begin{array}{l}93.5 \\
(88,100)\end{array}$ \\
\hline & & $\begin{array}{l}93.9 \\
(80,100)\end{array}$ & \\
\hline
\end{tabular}


Achillessenenrupturen im sport. In : Chapchal $G$, ed. Sportverletzung und Sportschaden. (pp. 75-77). Stuttgart: Ed. Georg Thieme

3. Hooker, C.H. (1963). Rupture of the tendo calcaneus.JBoneJoint Surg Br. 45, 360-363Inglis, A.E., Sculco, T.P. (1981). Surgical repair of the tendo of Achilles. Clin Orthop. 156, 160-169

4. Fitzgibbons, R.E., Hefferon, J. \& Hill J. (1993). Percutaneous Achilles tendon repair. Am J Sports Med. 21, 724-727
5. Saw, Y., Batzopoulos, V. \& Lim, A. et al. (1993). Early mobilization after operative repair of ruptured Achilles tendon. Injury. 24, 479-484

6. Poenaru, D.V., Matusz, P.L., Borza, I., Popa, C.A. \& Patrascu, J.M. (1997). Elemente de traumatologie. (pp. 9-36). Timisoara: Editura de Vest 